\title{
Molecule-based diagnosis of Apium graveolens allergy: is there a need to increase the current allergen panel?
}

\author{
G Gadermaier $^{1 *}$, E Vejvar ${ }^{1}$, P Briza ${ }^{1}$, K Hoffmann-Sommergruber ${ }^{2}$, W Hemmer $^{3}$, F Ferreira ${ }^{1}$ \\ From Food Allergy and Anaphylaxis Meeting (FAAM 2013) \\ Nice, France. 7-9 February 2013
}

\section{Background}

Consumption of celery (Apium graveolens) has the potential to trigger severe allergic reactions in sensitized patients and thus requires labeling on food products. The aim of the study was to determine the sensitization profile to celery allergens and to identify novel IgE binding proteins in this source.

\section{Methods}

Immunoblots were performed with extracts of celeriac and celery stalks using sera of $A$. graveolens sensitized patients from Austria $(n=23)$. Purified allergens were obtained from natural source (celeriac) or produced as recombinant proteins in $E$. coli and IgE reactivity to rApi g 1, rApi g 2, nApi g 5, and nApi g 6 was tested in ELISA. IgE cross-inhibition assays were performed with purified lipid transfer proteins (LTP). Celeriac extract was separated in 2D gel electrophoresis and IgE reactive spots were subjected to mass spectrometry-based analysis.

\section{Results}

Immunoblots showed distinct IgE reactive bands at 10 and $16 \mathrm{kDa}$ while broad reactivity was observed in the range between 25 and $100 \mathrm{kDa}$. IgE reactivity to Api g 1 , which is solely present in celery tuber and absent in stalks was $60 \%$ in A. graveolens sensitized patients. Fiftysix percent reacted to Api g 5 while $26-34 \%$ of patients were sensitized to Api g 6 (LTP2) and Api g 2 (LTP1) present in celery tuber and stalks, respectively. Interestingly, Api g 6 and Api g 2 sensitization pattern did not correlate and limited IgE cross-reactivity was observed.
Eighteen IgE reactive spots from celeriac extract were obtained in 2D gel electrophoresis. Mass analysis identified them as members of the fructose bisphosphate aldolase, malate dehydrogenase, GAPDH, triosephosphate isomerase, chitinase, and manganese superoxide dismutase families.

\section{Conclusion}

In vitro diagnosis using purified $A$. graveolens allergens is able to substitute extracts that might give rise to unspecific reactivity. In order to further enhance sensitivity and specificity, newly identified allergens should be produced and included in molecule-based diagnosis of celery allergy.

\section{Disclosure of interest}

None declared.

\section{Author details}

${ }^{1}$ Christian Doppler Laboratory for Allergy Diagnosis and Therapy, University of Salzburg, Salzburg, Austria. ${ }^{2}$ Department of Pathophysiology and Allergy Research, Medical University of Vienna, Vienna, Austria. ${ }^{3}$ FAZ-Floridsdorf Allergy Center, Vienna, Austria.

Published: 25 July 2013

doi:10.1186/2045-7022-3-S3-012

Cite this article as: Gadermaier et al: Molecule-based diagnosis of Apium graveolens allergy: is there a need to increase the current allergen panel? Clinical and Translational Allergy 2013 3(Suppl 3):012. 